\title{
Progression of Postprandial Blood Plasma Phospholipids Following Acute Intake of Different Dairy Matrices: A Randomized Crossover Trial
}

\author{
Rebekka Thøgersen ${ }^{1, *}$, Ida Emilie I. Lindahl ${ }^{1}$, Bekzod Khakimov ${ }^{2}{ }^{(\mathbb{D}}$, Louise Kjølbæk ${ }^{3}$, Klaus Juhl Jensen ${ }^{4}$, \\ Arne Astrup ${ }^{3}\left(\mathbb{O}\right.$, Marianne Hammershøj ${ }^{1}\left(\mathbb{D}\right.$, Anne Raben ${ }^{3,5}{ }^{-}$and Hanne Christine Bertram ${ }^{1}$ (D)
}

check for updates

Citation: Thøgersen, R.; Lindahl,

I.E.I.; Khakimov, B.; Kjølbæk, L.; Juhl Jensen, K.; Astrup, A.; Hammershøj, M.; Raben, A.; Bertram, H.C.

Progression of Postprandial Blood Plasma Phospholipids Following

Acute Intake of Different Dairy

Matrices: A Randomized Crossover Trial. Metabolites 2021, 11, 454. https://doi.org/10.3390/ metabo11070454

Academic Editor: Jose Manuel Lorenzo Rodriguez

Received: 3 June 2021

Accepted: 9 July 2021

Published: 14 July 2021

Publisher's Note: MDPI stays neutral with regard to jurisdictional claims in published maps and institutional affiliations.

Copyright: (c) 2021 by the authors. Licensee MDPI, Basel, Switzerland. This article is an open access article distributed under the terms and conditions of the Creative Commons Attribution (CC BY) license (https:/ / creativecommons.org/licenses/by/ $4.0 /)$.
1 Department of Food Science, Aarhus University, Agro Food Park 48, DK-8200 Aarhus N, Denmark; idaemilielindahl@gmail.com (I.E.I.L.); marianne.hammershoj@food.au.dk (M.H.); hannec.bertram@food.au.dk (H.C.B.)

2 Department of Food Science, University of Copenhagen, DK-1958 Frederiksberg C, Denmark; bzo@food.ku.dk

3 Department of Nutrition, Exercise and Sports, University of Copenhagen, DK-1958 Frederiksberg C, Denmark; louisekjoelbaek@nexs.ku.dk (L.K.); ara@novo.dk (A.A.); ara@nexs.ku.dk (A.R.)

4 Arla Foods Amba, DK-8200 Aarhus N, Denmark; kljes@arlafoods.com

5 Steno Diabetes Center Copenhagen, DK-2820 Gentofte, Denmark

* Correspondence: rebekka.thoegersen@food.au.dk

\begin{abstract}
Studies have indicated that the dairy matrix can affect postprandial responses of dairy products, but little is known about the effect on postprandial plasma phospholipid levels. This study investigated postprandial plasma phospholipid levels following consumption of four different dairy products that are similar in micro and macro nutrients, but different in texture and structure: cheddar cheese (Cheese), homogenized cheddar cheese (Hom. Cheese), micellar casein isolate with cream (MCI Drink) or a gel made from the MCI Drink (MCI Gel). The study was an acute randomized, crossover trial in human volunteers with four test days. Blood samples were collected during an $8 \mathrm{~h}$ postprandial period and the content of 53 plasma phospholipids was analysed using liquid chromatography-mass spectrometry (LC-MS). No meal-time interactions were revealed; however, for nine of the 53 phospholipids, a meal effect was found. Thus, the results indicated a lower plasma level of specific lyso-phosphatidylethanolamines (LPEs) and lyso-phosphatidylcholines (LPCs) following consumption of the MCI Gel compared to the MCI Drink and Hom. Cheese, which might be attributed to an effect of viscosity. However, further studies are needed in order to reveal more details on the effect of the dairy matrix on postprandial phospholipids.
\end{abstract}

Keywords: cheese; casein; phospholipidomics; food structure; milk phospholipids

\section{Introduction}

Dairy products are important sources of high-quality proteins, lipids, vitamins, and minerals (particularly calcium and phosphorus), and they constitute a significant contribution to diet quality because of their nutrient richness [1]. However, dairy products also contain considerable amounts of saturated fatty acid (SFA), which is commonly perceived as potentially harmful. Thus, consumption of high amounts of SFA is associated with increased blood LDL-cholesterol levels and risk of cardiovascular disease (CVD) [2]. Thus, while a reduction in SFA intake has been the cornerstone of dietary recommendations to decrease CVD risk, the validity of these recommendations has recently been questioned, as clear contradictions are evident [3]. Accordingly, studies have shown that the impact of SFA consumption on plasma lipids depends on the specific dietary source of SFA [4-6], and evidence exists that SFA contained in cheese matrices does not increase blood lipids as compared to other dairy matrices [7]. Although the exact causes for such different effects of 
SFA on plasma lipids are not yet fully understood, it is anticipated that the effect of the food matrix and physical/textural structures ('food matrix effect'), in which fat is incorporated and consumed, plays a pivotal role $[3,7,8]$. Apparently, intrinsic components present in the cheese matrix, such as protein, peptides, phospholipids derived from milk fat globule membrane (MFGM), calcium and phosphorous, are likely to influence the absorption of SFA in the human body [5,7].

Even though the exact underlying mechanisms behind this food matrix effect remain to be deciphered, some potential mechanisms have been proposed. Backed up by evidence revealing that calcium content impacts lipid absorption from dairy [9,10], it has been put forward that an underlying food matrix effect entails a reduction in fat bioaccessibility by calcium due to the formation of insoluble calcium soaps [11-13]. In addition, it has recently been hypothesized that milk phospholipids may bind cholesterol and thereby attenuate its absorption [14]. This hypothesis is supported by studies in animal models showing that milk phospholipids are reducing intestinal absorption of cholesterol [15], and thereby affecting plasma and hepatic lipid metabolism [16-18]. Recently, a 4-week intervention study with polar lipids derived from milk was shown to decrease plasma cholesterol, plasma triglycerides and Apolipoprotein B48 in overweight postmenopausal women [14]. Nevertheless, human studies focused on unravelling the role of phospholipids when consumed in the context of a whole dairy product are sparse. Therefore, the present study investigated the impact of a dairy matrix of four nutrient-matched meals of dairy products with different textural and structural properties on postprandial phospholipid absorption kinetics. Using a targeted liquid chromatography (LC) tandem mass spectrometry (MS) method previously developed for milk [19], quantification of approximately 50 glycerophospholipid and sphingolipid species was performed to investigate the presence of postprandial plasma phospholipids following consumption of the dairy products and to provide new knowledge about the effects of the dairy matrix on postprandial phospholipid status. The present study is part of the interdisciplinary DAIRYMAT project. The experimental design and primary outcome parameters are presented in Kjølbæk et al. [20] and a thorough characterization of the dairy products is provided in Schmidt et al. (2020) [21].

\section{Results}

LC-MS data are presented as arbitrary units; data correction was attained via normalization of the batch-corrected quantifier area of a given lipid according to the batch corrected quantifier of the corresponding internal standard.

Multivariate data analysis was used as an exploratory approach to investigate the phospholipid content in plasma samples during the postprandial period and a principal component analysis (PCA) model was constructed. The first two components explained $60 \%$ of the total variation. The PCA scores plot revealed an effect of time during the postprandial period (Supplementary Material Figure S1). The corresponding loadings plot revealed that plasma samples taken approximately two hours or more after consumption of the test meals were associated with higher content of PEs and PCs (Supplementary Material Figure S2).

Tables 1 and 2 and Supplementary Material Tables S1-S4 show the resulting mean values in plasma samples for LPE, LPC, CER, SM, PE and PC, respectively. No meal-time interactions were revealed for any of the phospholipids. For the following phospholipids, a meal effect was found: LPE 18:2 $(q=0.001)$, LPE 18:1 $(q<0.001)$, LPE 18:0 $(q=0.026)$, LPE 20:3 $(q=0.016)$, LPE 20:2 $(q<0.001)$, LPE 20:1 $(q=0.001)$ (Table 1), LPC 16:3 $(q=0.026)$, LPC 18:1 $(q=0.038)$, LPC 18:0 $(q=0.045)$ (Table 2$)$. The majority of the phospholipids showed no strong and systematic changes during the postprandial period and some remained almost constant during the 8-h period. Nevertheless, 31 out of the 53 investigated lipids showed an effect of time during the postprandial period $(q<0.05)$. 
Table 1. Relative level of lysophosphatidylethanolamine (LPE) in plasma samples.* Data were analyzed by repeated measures using a linear mixed model. Overall participant and within-visit participant differences were included as random factors and the analyses were adjusted for BMI, age and visit. $q$-values indicate FDR-adjusted $p$-values for meal-time interactions $\left(q_{\text {meal }}{ }^{*}\right.$ time $)$, the effect of meal $\left(q_{\text {meal }}\right)$ and the effect of time $\left(q_{\text {time }}\right)$. ${ }^{*}$ indicate significant differences $(q<0.05)$.

\begin{tabular}{|c|c|c|c|c|c|c|c|c|c|c|c|c|c|c|}
\hline Time (min) & 0 & 30 & 60 & 90 & 120 & 180 & 240 & 300 & 360 & 420 & 480 & $\mathrm{q}_{\text {meal * time }}$ & $\mathrm{q}_{\text {meal }}$ & $\mathbf{q}_{\text {time }}$ \\
\hline \multicolumn{15}{|l|}{ LPE 16:0 } \\
\hline Cheese & 0.018 & 0.018 & 0.015 & 0.015 & 0.017 & 0.016 & 0.017 & 0.014 & 0.017 & 0.015 & 0.014 & \multirow{4}{*}{0.79} & \multirow{4}{*}{0.29} & \multirow{4}{*}{0.11} \\
\hline Hom. Cheese & 0.026 & 0.021 & 0.019 & 0.018 & 0.017 & 0.021 & 0.018 & 0.024 & 0.018 & 0.021 & 0.019 & & & \\
\hline MCI Drink & 0.017 & 0.020 & 0.023 & 0.019 & 0.017 & 0.018 & 0.016 & 0.019 & 0.015 & 0.018 & 0.016 & & & \\
\hline MCI Gel & 0.015 & 0.019 & 0.016 & 0.017 & 0.018 & 0.014 & 0.014 & 0.016 & 0.016 & 0.015 & 0.014 & & & \\
\hline \multicolumn{15}{|l|}{ LPE 18:2 } \\
\hline Cheese & 0.335 & 0.412 & 0.303 & 0.295 & 0.317 & 0.309 & 0.296 & 0.281 & 0.314 & 0.286 & 0.332 & \multirow{4}{*}{0.69} & \multirow{4}{*}{$0.001 *$} & \multirow{4}{*}{0.30} \\
\hline Hom. Cheese & 0.419 & 0.278 & 0.367 & 0.307 & 0.282 & 0.308 & 0.283 & 0.316 & 0.354 & 0.300 & 0.358 & & & \\
\hline MCI Drink & 0.298 & 0.319 & 0.398 & 0.358 & 0.330 & 0.404 & 0.302 & 0.313 & 0.243 & 0.271 & 0.308 & & & \\
\hline MCI Gel & 0.263 & 0.288 & 0.279 & 0.290 & 0.286 & 0.246 & 0.251 & 0.250 & 0.224 & 0.268 & 0.268 & & & \\
\hline \multicolumn{15}{|l|}{ LPE 18:1 } \\
\hline Cheese & 0.824 & 0.846 & 0.650 & 0.726 & 0.747 & 0.692 & 0.675 & 0.596 & 0.711 & 0.607 & 0.598 & \multirow{4}{*}{0.79} & \multirow{4}{*}{$<0.001 *$} & \multirow{4}{*}{$<0.001 *$} \\
\hline Hom. Cheese & 0.959 & 0.807 & 0.896 & 0.795 & 0.694 & 0.820 & 0.722 & 0.774 & 0.750 & 0.730 & 0.823 & & & \\
\hline MCI Drink & 0.800 & 0.845 & 0.969 & 0.868 & 0.794 & 0.787 & 0.675 & 0.715 & 0.553 & 0.698 & 0.727 & & & \\
\hline MCI Gel & 0.633 & 0.787 & 0.692 & 0.731 & 0.659 & 0.535 & 0.488 & 0.522 & 0.501 & 0.553 & 0.587 & & & \\
\hline \multicolumn{15}{|l|}{ LPE 18:0 } \\
\hline Cheese & 1.780 & 1.878 & 1.498 & 1.636 & 1.742 & 1.615 & 1.564 & 1.361 & 1.642 & 1.371 & 1.329 & \multirow{4}{*}{0.79} & \multirow{4}{*}{$0.026^{*}$} & \multirow{4}{*}{0.001 * } \\
\hline Hom. Cheese & 2.141 & 1.723 & 1.923 & 1.674 & 1.613 & 1.838 & 1.622 & 1.819 & 1.733 & 1.638 & 1.814 & & & \\
\hline MCI Drink & 1.714 & 1.856 & 2.139 & 1.953 & 1.793 & 1.762 & 1.561 & 1.665 & 1.256 & 1.520 & 1.572 & & & \\
\hline MCI Gel & 1.499 & 1.792 & 1.590 & 1.690 & 1.641 & 1.347 & 1.254 & 1.339 & 1.229 & 1.329 & 1.365 & & & \\
\hline \multicolumn{15}{|l|}{ LPE 20:3 } \\
\hline Cheese & 0.105 & 0.121 & 0.091 & 0.102 & 0.096 & 0.097 & 0.095 & 0.105 & 0.116 & 0.098 & 0.120 & \multirow{4}{*}{0.79} & \multirow{4}{*}{$0.016^{*}$} & \multirow{4}{*}{0.010 * } \\
\hline Hom. Cheese & 0.126 & 0.093 & 0.129 & 0.107 & 0.090 & 0.103 & 0.099 & 0.107 & 0.119 & 0.113 & 0.137 & & & \\
\hline MCI Drink & 0.111 & 0.106 & 0.122 & 0.107 & 0.103 & 0.111 & 0.108 & 0.109 & 0.090 & 0.116 & 0.124 & & & \\
\hline MCI Gel & 0.090 & 0.093 & 0.090 & 0.104 & 0.094 & 0.078 & 0.083 & 0.090 & 0.085 & 0.106 & 0.104 & & & \\
\hline \multicolumn{15}{|l|}{ LPE 20:2 } \\
\hline Cheese & 0.103 & 0.109 & 0.077 & 0.089 & 0.085 & 0.085 & 0.082 & 0.070 & 0.088 & 0.073 & 0.075 & & & \\
\hline Hom. Cheese & 0.124 & 0.102 & 0.108 & 0.095 & 0.086 & 0.096 & 0.087 & 0.100 & 0.092 & 0.090 & 0.105 & 0.79 & $<0.001 *$ & $<0.001 *$ \\
\hline MCI Drink & 0.098 & 0.109 & 0.116 & 0.108 & 0.096 & 0.092 & 0.081 & 0.083 & 0.062 & 0.091 & 0.095 & & & \\
\hline MCI Gel & 0.076 & 0.094 & 0.080 & 0.091 & 0.080 & 0.061 & 0.056 & 0.067 & 0.063 & 0.073 & 0.075 & & & \\
\hline LPE 20:1 & & & & & & & & & & & & & & \\
\hline Cheese & 1.332 & 1.384 & 1.015 & 1.121 & 1.188 & 1.077 & 1.034 & 0.968 & 1.197 & 1.019 & 1.057 & & & \\
\hline Hom. Cheese & 1.533 & 1.277 & 1.353 & 1.165 & 1.120 & 1.201 & 1.117 & 1.167 & 1.228 & 1.163 & 1.370 & 0.79 & 0,001 * & $<0,001$ * \\
\hline MCI Drink & 1.266 & 1.393 & 1.497 & 1.385 & 1.215 & 1.245 & 1.052 & 1.206 & 0.891 & 1.190 & 1.264 & & & \\
\hline MCI Gel & 1.066 & 1.222 & 1.027 & 1.163 & 1.036 & 0.819 & 0.795 & 0.866 & 0.830 & 0.972 & 1.024 & & & \\
\hline LPE 22:6 & & & & & & & & & & & & & & \\
\hline Cheese & 1.108 & 1.227 & 0.962 & 1.017 & 1.130 & 0.967 & 1.063 & 0.952 & 1.140 & 0.984 & 1.038 & & & \\
\hline Hom. Cheese & 1.295 & 1.011 & 1.155 & 1.120 & 0.982 & 1.110 & 1.014 & 1.246 & 1.096 & 1.106 & 1.204 & 079 & 007 & 030 \\
\hline MCI Drink & 1.166 & 1.183 & 1.279 & 1.187 & 1.190 & 1.175 & 1.106 & 1.088 & 0.979 & 1.080 & 1.165 & 0.1 & ת. & 80 \\
\hline MCI Gel & 1.033 & 1.148 & 0.966 & 1.024 & 0.979 & 0.949 & 0.904 & 0.991 & 0.842 & 0.946 & 0.955 & & & \\
\hline
\end{tabular}

* Fatty acid moiety is shown with number of carbons followed by the number of double bond of carbons in the fatty acid chain. 
Table 2. Relative level of lysophosphatidylcholine (LPC) in plasma samples.* Data were analyzed by repeated measures using a linear mixed model. Overall participant and within-visit participant differences were included as random factors and the analyses were adjusted for BMI, age and visit. $q$-values indicate FDR-adjusted $p$-values for meal-time interactions $\left(\mathrm{q}_{\text {meal }}\right.$ time $)$, the effect of meal $\left(\mathrm{q}_{\text {meal }}\right)$ and the effect of time $\left(\mathrm{q}_{\text {time }}\right)$. ${ }^{*}$ indicate significant differences $(q<0.05)$.

\begin{tabular}{|c|c|c|c|c|c|c|c|c|c|c|c|c|c|c|}
\hline Time (min) & 0 & 30 & 60 & 90 & 120 & 180 & 240 & 300 & 360 & 420 & 480 & $\mathrm{q}_{\text {meal * time }}$ & $\mathrm{q}_{\text {meal }}$ & $\mathrm{q}_{\text {time }}$ \\
\hline \multicolumn{15}{|l|}{ LPC 16:3 } \\
\hline Cheese & 99.428 & 112.408 & 87.591 & 94.724 & 102.710 & 88.845 & 98.695 & 86.458 & 99.880 & 91.332 & 92.618 & \multirow{4}{*}{0.79} & \multirow{4}{*}{$0.026^{*}$} & \multirow{4}{*}{0.17} \\
\hline Hom. Cheese & 111.998 & 90.966 & 106.467 & 97.927 & 89.453 & 100.357 & 89.250 & 111.806 & 97.412 & 93.725 & 107.311 & & & \\
\hline MCI Drink & 99.916 & 104.535 & 109.441 & 104.144 & 104.554 & 99.942 & 96.144 & 94.973 & 82.139 & 91.793 & 97.931 & & & \\
\hline MCI Gel & 91.769 & 103.383 & 83.215 & 88.737 & 89.650 & 82.000 & 75.879 & 84.615 & 74.239 & 79.843 & 86.073 & & & \\
\hline \multicolumn{15}{|l|}{ LPC 18:4 } \\
\hline Cheese & 0.192 & 0.265 & 0.172 & 0.185 & 0.196 & 0.186 & 0.269 & 0.175 & 0.240 & 0.285 & 0.207 & \multirow{4}{*}{0.69} & \multirow{4}{*}{0.56} & \multirow{4}{*}{0.031 * } \\
\hline Hom. Cheese & 0.240 & 0.150 & 0.133 & 0.202 & 0.244 & 0.223 & 0.194 & 0.294 & 0.211 & 0.247 & 0.295 & & & \\
\hline MCI Drink & 0.225 & 0.238 & 0.222 & 0.222 & 0.229 & 0.155 & 0.254 & 0.198 & 0.178 & 0.245 & 0.265 & & & \\
\hline MCI Gel & 0.185 & 0.242 & 0.141 & 0.166 & 0.164 & 0.186 & 0.168 & 0.253 & 0.206 & 0.200 & 0.223 & & & \\
\hline \multicolumn{15}{|l|}{ LPC 18:1 } \\
\hline Cheese & 37.100 & 40.785 & 31.934 & 32.954 & 34.604 & 31.160 & 35.798 & 32.833 & 40.197 & 37.388 & 39.552 & \multirow{4}{*}{0.79} & \multirow{4}{*}{$0.038 *$} & \multirow{4}{*}{0.005 * } \\
\hline Hom. Cheese & 43.961 & 32.096 & 39.357 & 34.708 & 33.136 & 36.201 & 33.256 & 39.231 & 39.143 & 39.298 & 46.104 & & & \\
\hline MCI Drink & 37.529 & 38.037 & 40.413 & 38.786 & 37.626 & 38.473 & 35.659 & 37.146 & 32.123 & 40.174 & 42.776 & & & \\
\hline MCI Gel & 32.524 & 35.282 & 30.447 & 33.027 & 31.176 & 29.543 & 27.902 & 32.784 & 29.748 & 35.714 & 36.609 & & & \\
\hline \multicolumn{15}{|l|}{ LPC 18:0 } \\
\hline Cheese & 42.735 & 47.521 & 36.862 & 39.382 & 43.097 & 37.804 & 41.202 & 37.245 & 43.150 & 38.534 & 40.294 & \multirow{4}{*}{0.79} & \multirow{4}{*}{$0.045^{*}$} & \multirow{4}{*}{0.30} \\
\hline Hom. Cheese & 50.650 & 39.148 & 45.818 & 41.800 & 38.243 & 44.158 & 39.142 & 46.650 & 42.214 & 43.289 & 46.385 & & & \\
\hline MCI Drink & 45.162 & 45.427 & 49.535 & 46.238 & 45.999 & 47.192 & 42.386 & 43.521 & 37.251 & 41.634 & 44.870 & & & \\
\hline MCI Gel & 39.870 & 43.515 & 37.074 & 39.537 & 39.408 & 36.150 & 35.096 & 37.522 & 32.041 & 36.311 & 37.531 & & & \\
\hline \multicolumn{15}{|l|}{ LPC 20:0 } \\
\hline Cheese & 0.436 & 0.388 & 0.489 & 0.560 & 0.883 & 0.606 & 0.670 & 0.632 & 0.751 & 0.602 & 0.762 & \multirow{4}{*}{0.91} & \multirow{4}{*}{0.80} & \multirow{4}{*}{0.049 * } \\
\hline Hom. Cheese & 0.516 & 0.426 & 0.544 & 0.572 & 0.576 & 0.760 & 0.732 & 0.583 & 0.679 & 0.763 & 0.655 & & & \\
\hline MCI Drink & 0.442 & 0.418 & 0.550 & 0.463 & 0.569 & 0.897 & 0.502 & 0.722 & 0.588 & 0.671 & 0.633 & & & \\
\hline MCI Gel & 0.371 & 0.399 & 0.664 & 0.677 & 0.622 & 0.508 & 0.543 & 0.556 & 0.653 & 0.507 & 0.562 & & & \\
\hline \multicolumn{15}{|l|}{ LPC 22:0 } \\
\hline Cheese & 0.00014 & 0.00008 & 0.00007 & 0.00007 & 0.00008 & 0.00007 & 0.00006 & 0.00007 & 0.00013 & 0.00008 & 0.00010 & \multirow{4}{*}{0.83} & \multirow{4}{*}{0.45} & \multirow{4}{*}{0.48} \\
\hline Hom. Cheese & 0.00008 & 0.00008 & 0.00007 & 0.00004 & 0.00005 & 0.00009 & 0.00006 & 0.00007 & 0.00009 & 0.00008 & 0.00011 & & & \\
\hline MCI Drink & 0.00007 & 0.00006 & 0.00009 & 0.00006 & 0.00009 & 0.00009 & 0.00007 & 0.00006 & 0.00008 & 0.00006 & 0.00009 & & & \\
\hline MCI Gel & 0.00005 & 0.00004 & 0.00007 & 0.00011 & 0.00007 & 0.00007 & 0.00008 & 0.00008 & 0.00007 & 0.00005 & 0.00006 & & & \\
\hline LPC 24:0 & & & & & & & & & & & & & & \\
\hline Cheese & 0.586 & 0.539 & 0.647 & 0.713 & 0.932 & 0.613 & 0.501 & 0.958 & 0.636 & 0.636 & 0.772 & & & \\
\hline Hom. Cheese & 0.851 & 0.627 & 0.898 & 0.964 & 0.504 & 0.666 & 0.553 & 0.571 & 0.625 & 0.626 & 0.808 & 089 & 076 & 0.48 \\
\hline MCI Drink & 0.793 & 0.561 & 0.730 & 0.695 & 0.883 & 0.803 & 0.729 & 0.832 & 0.753 & 0.683 & 0.824 & & & \\
\hline MCI Gel & 0.561 & 0.533 & 0.838 & 0.701 & 0.592 & 0.556 & 0.719 & 0.411 & 0.582 & 0.626 & 0.533 & & & \\
\hline
\end{tabular}

* Fatty acid moiety is shown with number of carbons followed by the number of double bond of carbons in the fatty acid chain.

For the nine phospholipids showing an effect of meal, differences between the treatment groups were evaluated by Tukey's all-pairwise comparison. For all the nine phospholipids, significant differences were found between consumption of MCI Drink and MCI Gel and between consumption of Hom. Cheese and MCI Gel. Generally, consumption of MCI Gel appeared to result in the lowest postprandial level of the nine phospholipids and, for all of the nine phospholipids, a significant difference was found between consumption of MCI Gel and MCI Drink and between consumption of MCI Gel and Hom. Cheese (Figures 1, 2 and S3). For LPE 18:2 and LPC 16:3, significant differences were also found between consumption of cheese and MCI Gel (Figures S3.1 and S3.2). Likewise, for LPE 18:1 and LPE 20:2, significant differences were found between consumption of cheese and Hom. Cheese (Figures 1 and S3.4). 


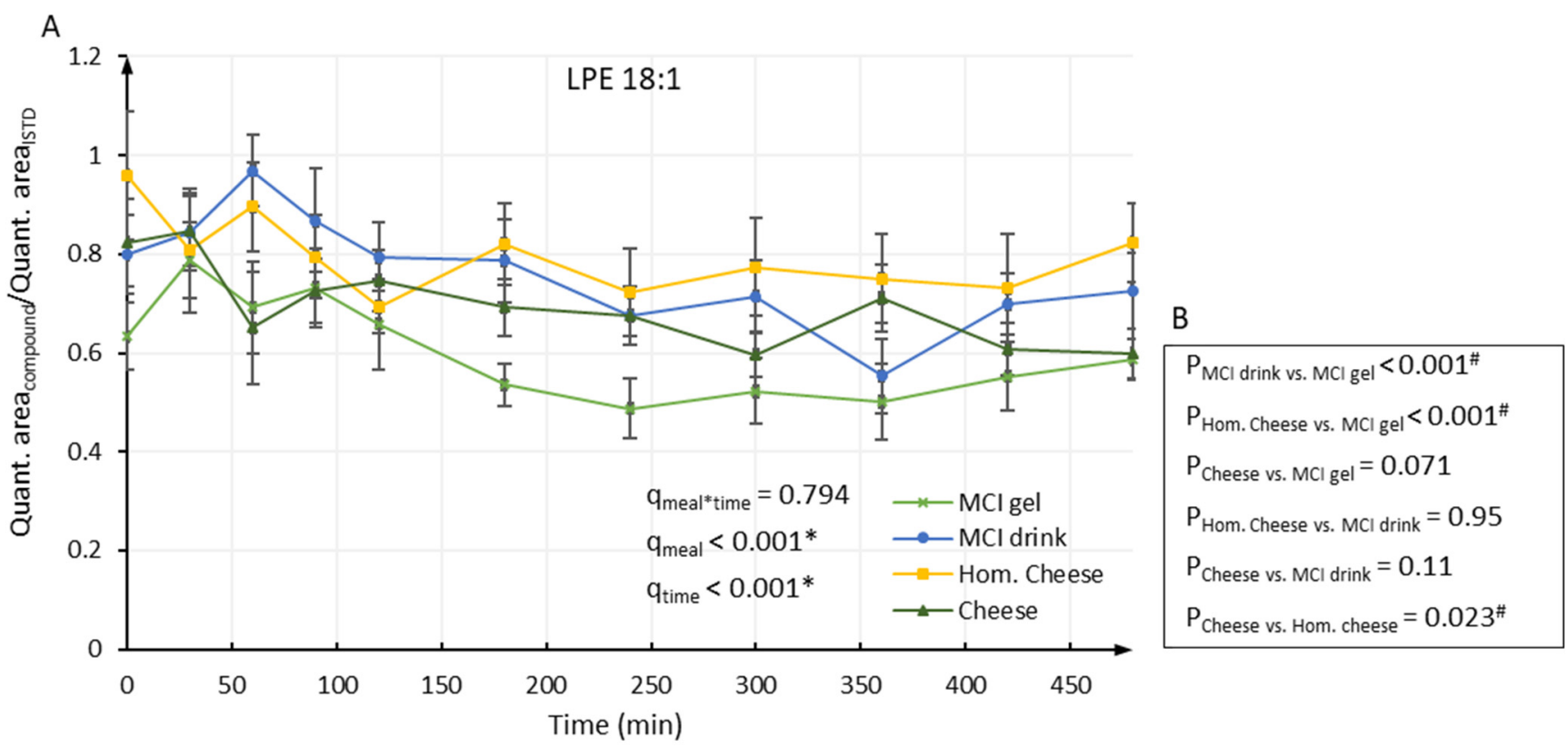

Figure 1. (A) Relative level of LPE 18:1 in plasma samples. Data were analyzed by repeated measures using a linear mixed model. Overall participant and within-visit participant differences were included as random factors and the analyses were adjusted for BMI, age and visit. $q$-values indicate FDR-adjusted $p$-values for meal-time interactions $\left(q_{\text {meal }}^{*}\right.$ time $)$, the effect of meal $\left(q_{\text {meal }}\right)$ and the effect of time $\left(q_{\text {time }}\right)$. * indicate significant differences $(q<0.05)$. $(\mathbf{B}) p$-values obtained from Tukey's all-pairwise comparison conducted when a significant meal effect was observed in repeated measures analysis. ${ }^{\#}$ indicate significant differences $(p<0.05)$.

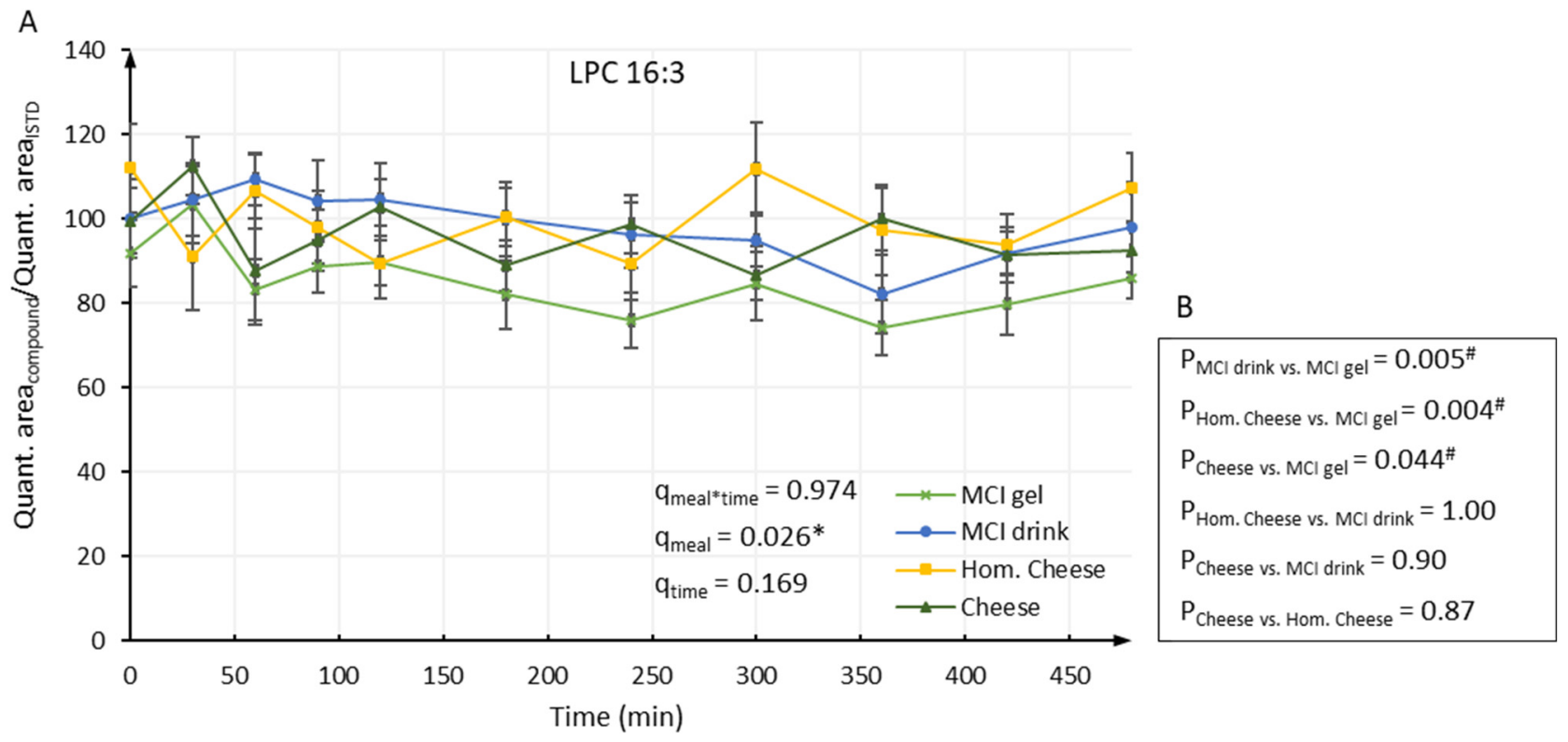

Figure 2. (A) Relative level of LPC 16:3 in plasma samples. Data were analyzed by repeated measures using a linear mixed model. Overall participant and within-visit participant differences were included as random factors and the analyses were adjusted for BMI, age and visit. $q$-values indicate FDR-adjusted $p$-values for meal-time interactions $\left(\mathrm{q}_{\text {meal }} *\right.$ time $)$, the effect of meal $\left(q_{\text {meal }}\right)$ and the effect of time $\left(q_{\text {time }}\right)$. * indicate significant differences $(q<0.05)$. (B) $p$-values obtained from Tukey's all-pairwise comparison conducted when a significant meal effect was observed in repeated measures analysis. ${ }^{\#}$ indicate significant differences $(p<0.05)$. 


\section{Discussion}

In the present study, no meal-time interactions were revealed for any of the analysed phospholipids. However, lower phospholipid levels were found after consumption of the MCI gel compared to the MCI drink and the Hom. cheese in nine of the 53 investigated phospholipids.

Increasing evidence indicates that not only the single nutrients, but also the complex structure of a given food can influence its health effects following consumption. Thus, the nature of the food matrix may play an important role by affecting the absorption and digestion of a given food [7]. In fact, previous studies found that the dairy matrix can affect the postprandial response and nutritional properties of dairy products [6,22]. Milk contains phospholipids, which are located within the MFGM, the trilayered membrane surrounding the fat globules. Dairy phospholipids play an important role in the emulsification of fat in milk, but their impact on human health has also received attention $[23,24]$. Hence, studies have indicated potential health beneficial effects of dietary phospholipid consumption including decreased cholesterol absorption, suppressed intestinal inflammation, and modulation of the gut microbiota $[14,23,25,26]$. Previous studies have shown that the postprandial blood phospholipid status in humans can be affected by diet $[27,28]$, and a recent study also revealed that the postprandial changes in blood phospholipid are perturbed in people with metabolic syndrome [29]. Meikle et al. (2015) investigated the consumption of dairy fat and soy oil and found that the source of dietary fat affected the postprandial plasma phospholipid concentrations in men [28]. Thus, a few studies have investigated the effect of dietary sources on postprandial blood phospholipid status, but little is known about the effect of the dairy matrix on postprandial blood phospholipids levels. In the present study, the majority of the 53 phospholipids examined in plasma showed minor concentration changes during the postprandial period and some remained steady in level. For the majority of the analysed phospholipids in the present study, no meal effect was found. These results are in accordance with those of Weiland et al. (2016), who found that milk phospholipid consumption did not affect the overall plasma phospholipid levels [30]. However, in the present study, for nine of the 53 investigated phospholipids, an effect of meal was observed. Thus, a meal effect was found for six out of eight measured LPEs and three out of seven measured LPCs. Generally, for these nine phospholipids, lower levels were found after consumption of the MCI Gel compared to the MCI Drink and the Hom. Cheese. Meikle et al. (2015) also found that consumption of a dairy meal differently affected postprandial lysophospholipid levels. Thus, dairy meal consumption increased postprandial levels of total LPEs and lysophosphatidylinositol, whereas no changes were observed for postprandial levels of total LPC [28]. When consumed, phospholipids are hydrolyzed in the intestine, resulting in the formation of lysophospholipids and free fatty acids [31]. Hence, in the present study, the metabolism of specific phospholipids might have been differently affected, leading to different effects on specific LPEs and LPCs.

The observed meal effects were not reflected in the phospholipid content of the analysed meals, which did not differ between the meals (data not shown). The lower level of phospholipids following consumption of the MCI Gel might be ascribed to a number of different factors. Comparing the MCI Gel and the MCI Drink, the MCI Gel had an increased viscosity prior to digestion [21]. Previous studies have indicated that an increased viscosity can slow gastric emptying rate [32,33], which could possibly explain the lower level of phospholipids in plasma following the MCI Gel. In addition, the size of the fat droplets in the dairy products might play a role in postprandial phospholipid response in the present study. Analyses of the dairy products revealed that the MCI Gel had a significantly larger fat droplet size compared to the MCI Drink and Hom. Cheese prior to digestion [21]. In fact, it has previously been found that larger fat droplets sizes can result in a slower digestion [34]. However, during in vitro digestion, the particle size of the products changed. The MCI Gel had the largest volume $\mathrm{D}(4.3)$ weighted diameter values during the gastric digestion, while during the intestinal digestion, the $\mathrm{D}(4.3)$ increased for the Cheese to a level significantly $(p<0.01)$ larger than for the three other products [21]. For surface $\mathrm{D}(3.2)$ 
weighed diameters, on the other hand, Hom. Cheese had the significantly highest level during gastric digestion, and again the Cheese was shown to have the largest particle sizes in the intestinal digestion phase [21]. In addition to fat droplet size, it could be speculated that the stability and intactness of the MFGM may also affect the postprandial phospholipid status. Under physiological $\mathrm{pH}$ and temperature, the MFGM is regarded as relatively stable, but various factors including homogenization and changes in temperature, salt and $\mathrm{pH}$ can induce destabilization [35]. Therefore, since the majority of the milk phospholipids are expected to be located within the MFGM, variations in the mentioned factors among the dairy products could potentially play a role in the observed meal effects. However, further studies are required to identify possible effects of the stability of the MGFM on phospholipid absorption.

The present study has some limitations. As a normalization of the LC-MS data was necessary to overcome between-batch variations resulting from drifts during data acquisition, relative concentrations of phospholipids are reported. While this quantitation allows a comparison of different meals, subjects etc., a direct comparison of the reported concentrations to other studies is not possible. In terms of mechanistic data interpretation, the phospholipid analyses were based on unfractionated blood plasma. Fractionation into different lipoproteins and chylomicrons could be expected to provide additional information in relation to the exogenous or endogenous origin of the phospholipids.

Differences in the amount of phospholipids present in the meals may impact the post prandial phospholipid profile, and another limitation with the present study is the fact that the amount of phospholipids in the meals was not quantified. Neither was it characterized how the processing, i.e., the homogenization of the cheese, affected the phospholipid composition. However, our main goal was to examine differences in the postprandial kinetics as functions of the different meal products' textures and structures.

In conclusion, no meal-time interactions were revealed; however, for nine of the 53 phospholipids, a meal effect was found. The results indicated a lower plasma level of specific LPEs and LPCs following consumption of the MCI Gel compared to the MCI Drink and Hom. Cheese. However, further studies are needed in order to reveal the detailed effect of the dairy matrix on postprandial phospholipids.

\section{Materials and Methods}

\subsection{Dairy Products}

The dairy products differed in structural (solid, semi-solid, and liquid) and textural integrity. Briefly, a commercial full-fat cheddar cheese (Cheese) (Arla Foods Ltd., Leeds, UK) with native milk fat globules and an intact protein network constituted the solid cheese matrix. A semi-solid cheese matrix with homogenized milk fat globules and partial loss of the protein network (Hom. Cheese) was produced from the commercial full-fat cheese through homogenization. A solution of micellar casein isolate (MCI) with cream and salt (MCI Drink), containing native milk fat globules but void of a protein network, constituted the liquid cheese matrix. Finally, a gel was produced based on the MCI Drink mixed with glucono- $\delta$-lactone to form a semi-solid matrix (MCI Gel) with a gelled protein network and native milk fat globules. In order to obtain similar macro and micro nutrient content and ensure an overall energy consumption, the dairy products were served with bread and water [20].

\subsection{Test Participants and Study Design}

The study was an acute, cross-over intervention study consisting of four test days (Figure 3) conducted from September 2018 to March 2019 at the Department of Nutrition, Exercise and Sports, University of Copenhagen, Denmark. Co-primary outcomes (postprandrial triglycerides and Apolipoprotein B-48) are reported in Kjølbæk et al. [20] and in the present study, data from the analysis of postprandial plasma phospholipids are reported. Participants were recruited using announcements in Danish newspapers and various internet platforms, and a total of 25 men were enrolled. The inclusion criteria 
were as follows: healthy men (between 18 and 40 years), non-smoker, body mass index (BMI): $18.5-24.9 \mathrm{~kg} / \mathrm{m}^{2}$, hemoglobin concentration $\geq 8.4 \mathrm{mmol} / \mathrm{L}$. Participants meeting the exclusion criteria were omitted [20]. Participants who completed a minimum of two test days $(n=21)$ were included in the analyses, and 18 participants completed all four test days. For standardization, the study participants were instructed to avoid alcohol, medication and strenuous physical activity $48 \mathrm{~h}$ prior to the test day. Study participants consumed a standardized meal on the evening prior to the test day and were instructed to finish the meal before 10 in the evening, after which they had to fast until receiving the test meal the following day. For all participants, the order in which they received the four test meals was randomized. On each test day, participants were weighed upon arrival and fasting blood samples were drawn (time $0 \mathrm{~min}$.), after which the participants received a test meal (4.7 MJ) that included one of the four dairy products, and $1.5 \mathrm{~g}$ paracetamol, which was to be consumed within $15 \mathrm{~min}$. During the $8 \mathrm{~h}$ period, participants drank two glasses of water. Postprandial blood samples were drawn at time points 30, 60, 90, 120, 180, 240, $300,360,420$ and $480 \mathrm{~min}$. and plasma was collected and stored at $-80^{\circ} \mathrm{C}$ until analysis.

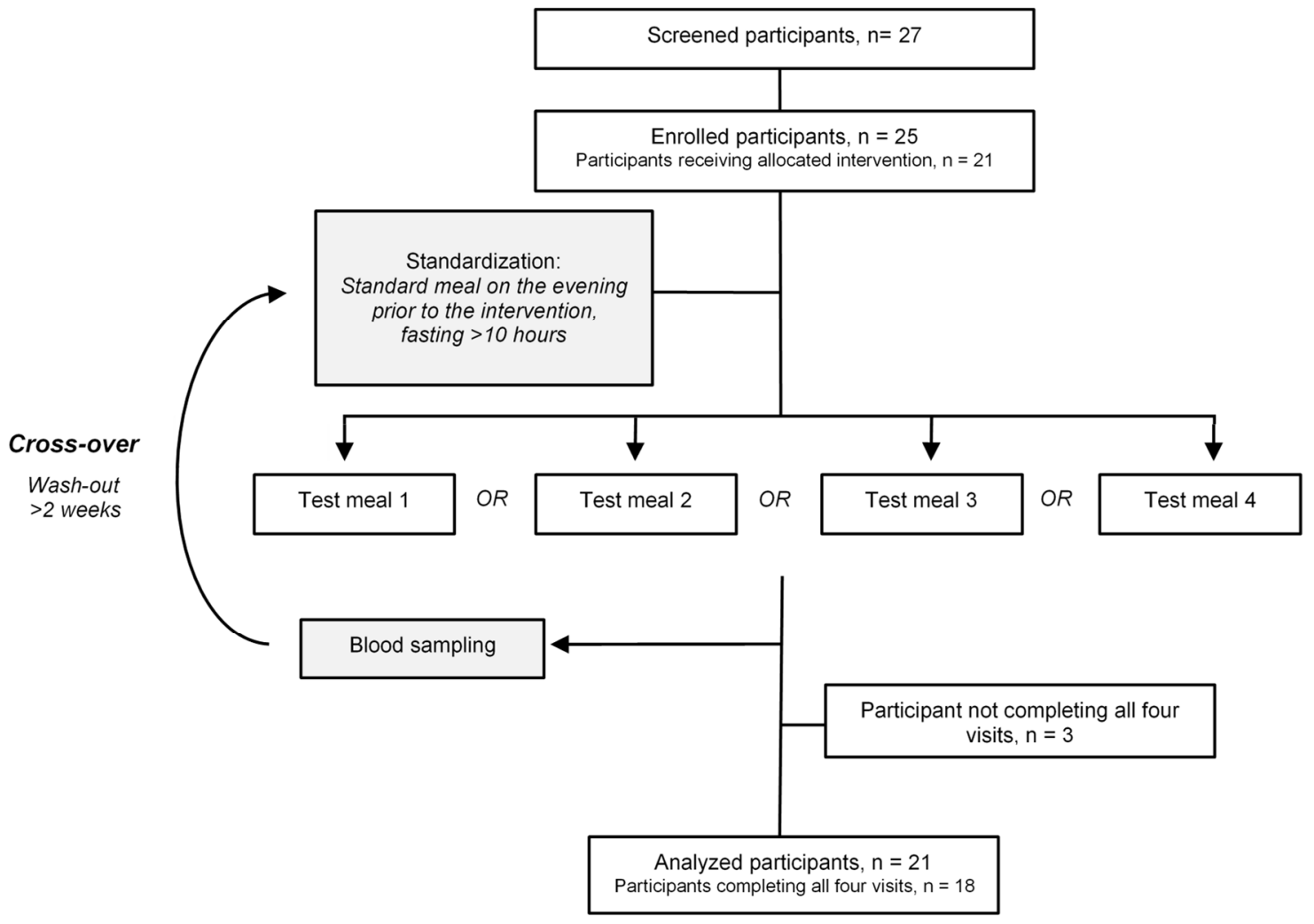

Figure 3. Study design.

\subsection{Materials}

The lipid standards 3-sn-phosphatidylethanolamine (from bovine brain, $\geq 98 \%$ ) and L- $\alpha$-phosphatidylcholine (from egg yolk, $\geq 99 \%$ ) were purchased from Merck KGaA (Darmstadt, Germany), sphingomyelin (from bovine buttermilk, $>98 \%$ ), lyso-phosphatidylethanolamine (from bovine brain, $>98 \%$ ) and Ceramide (mixture, $>98 \%$ ) were purchased from Larodan (Solna, Sweden), and L- $\alpha$-Lysophosphatidylcholine (from egg yolk, $>98 \%$ ) was purchased from Santa Cruz Biotechnology (Dallas, TX, USA). The deuterated internal standard SPLASH ${ }^{\circledR}$ LIPIDOMIX ${ }^{\circledR}$ Mass Spec Standard (Avanti Polar Lipids, Alabaster, AL, USA) was purchased from Merck KGaA (Darmstadt, Germany). Methanol (HiPerSolv CHROMANORM $^{\circledR}$, LC-MS grade, $\geq 99.9 \%$ ), acetonitrile (HiPerSolv CHROMANORM $^{\circledR}$, LC-MS grade, $\geq 99.9 \%$ ) and water (HiPerSolv CHROMANORM ${ }^{\circledR}$ LC-MS grade) were purchased from VWR (Søborg, Denmark), and chloroform ( $\geq 99.9 \%$, LC-MS grade) was 
purchased from Rathburn Chemicals Ltd. (Walkerburn, Scotland). Ammonium formate (LC-MS grade, 99.0\%) was purchased from Sigma-Aldrich (St. Louis, MO, USA), and formic acid (LC-MS LiChropur ${ }^{\circledR}, 98-100 \%$ ) was purchased from Merck KGaA (Darmstadt, Germany). A water purification system (Holm \& Halby, Brøndby, Denmark) was used to prepare ultrapure $(18.2 \mathrm{M} \Omega$ ) water.

\subsection{Extraction of Plasma Lipids}

Plasma samples $(n=880)$ were randomized prior to extraction. Extraction of polar lipids was based on a modified version of the Bligh and Dyer method [36]. Samples were thawed on ice and whirl pooled, after which $100 \mu \mathrm{L}$ plasma was transferred to a centrifuge tube. To the centrifuge tube, $1 \mu \mathrm{L}$ deuterated internal standard was added and briefly mixed on a vortex mixer, followed by the addition of $737 \mu \mathrm{L}$ ice cold methanol:water solution $(v / v 2: 0.8)$. The samples were briefly mixed on a vortex mixer, after which $263 \mu \mathrm{L}$ ice cold chloroform was added and followed by vortex mixing. After addition of the extraction solvents, the centrifuge tubes were centrifuged at $20,000 \times g$ for $10 \mathrm{~min}$ at $4{ }^{\circ} \mathrm{C}$ and the organic and aqueous phases were transferred to a new centrifuge tube, discarding the interphase. Solvents were vaporized at $30{ }^{\circ} \mathrm{C}$ under vacuum, and the resulting lipid extracts were suspended in $1000 \mu \mathrm{L}$ ice cold methanol. Finally, the samples were centrifuged at $20,000 \times \mathrm{g}$ for $10 \mathrm{~min}$ at $4{ }^{\circ} \mathrm{C}$ and the supernatants were transferred to new centrifuge tubes and stored at $-80{ }^{\circ} \mathrm{C}$ until analysis. Prior to analysis, the samples were further centrifuged $\left(20,000 \times g\right.$ for $10 \mathrm{~min}$ at $\left.4^{\circ} \mathrm{C}\right)$ to remove insoluble particles and the supernatant transferred to 96 well plates.

\subsection{Extraction of Dairy Lipids}

For preparation of the dairy products, samples $(n=4)$ were prepared in technical triplicates. The samples were frozen at $-18{ }^{\circ} \mathrm{C}$ and lyophilized. After lyophilization, samples were transferred to a mortar and ground. Liquid nitrogen was added to the mortar to ensure homogenous samples. To a centrifuge tube, $20 \mathrm{mg}$ sample was transferred and lipids were extracted using the same method as for the plasma samples.

\subsection{Standards and Calibration Curves}

Stock solutions of polar lipid standards, one for each of the six lipid classes, were prepared in chloroform (phosphatidylcholine (PC), phosphatidylethanolamine (PE), lysophosphatidylcholine (LPC), ceramide (CER) and sphingomyelin (SM): $2.5 \mathrm{~g} / \mathrm{L}$, lysophosphatidylethanolamine (LPE): $1 \mathrm{~g} / \mathrm{L})$. For calibration, different concentration levels were constructed by dilution of the stock solutions in methanol (PC: $6.7 \times 10^{-6}-1 \mathrm{~g} / \mathrm{L}, \mathrm{PE}: 3.4 \times 10^{-6}-0.5 \mathrm{~g} / \mathrm{L}$, LPC and SM: 2.7-0.4 g/L, LPE: $1.1 \times 10^{-7}-1.6 \times 10^{-2} \mathrm{~g} / \mathrm{L}$, Cer: $\left.6.7 \times 10^{-7}-0.1 \mathrm{~g} / \mathrm{L}\right)$. Deuterated internal standard $(1 \mu \mathrm{L})$ was added to each calibration level. A total of three deuterated internal standards were used; deuPC was used to quantitate PC and PE, deuLPC was used for LPC and LPE, and deuSM was used for SM and Cer. To monitor batch variation, two concentration levels of mixed lipid standards in methanol were used for the quality controls (QC). Stock solutions, calibration levels and QCs were stored in glass tubes at $-20{ }^{\circ} \mathrm{C}$.

\subsection{Liquid Chromatography Tandem Mass Spectrometry}

The method for semi-quantitative analysis of polar lipids (ng/ $\mu \mathrm{L})$ was based on a LC-ESI-MS/MS method as previously described [19], with few modifications. Briefly, the LC-MS system consisted of an Agilent 1290 Ultra Performance Liquid Chromatography coupled to an Agilent 6495 Triple Quadrupole Mass Spectrometer (Agilent Technologies, Palo Alto, CA, USA) operated in ESI positive mode. Eluent A consisted of water with $10 \mathrm{mM}$ ammonium formate (adjusted to $\mathrm{pH} 3$ ) and eluent B consisted of acetonitrile. Ninety-six-well plates containing the extracted samples were placed in the multisampler at $4{ }^{\circ} \mathrm{C}$ and $5 \mu \mathrm{L}$ was injected onto an ACQUITY UPLC BEH HILIC Column $(1.7 \mu \mathrm{m}, 2.1 \times 100 \mathrm{~mm})$ coupled with an ACQUITY UPLC BEH HILIC VanGuard Pre-column $(1.7 \mu \mathrm{m}, 2.1 \mathrm{~mm} \times 5 \mathrm{~mm}$, Wa- 
ters Corporation, Milford, MA, USA). A flowrate of $0.500 \mathrm{~mL} / \mathrm{min}$ and column temperature of $25^{\circ} \mathrm{C}$ was used. The percentage of eluent $\mathrm{B}$ used for chromatographic separation was as follows: $0.00-0.10 \mathrm{~min}$ : $95 \%$; $0.10-20 \mathrm{~min}: 95-80 \%$; $20-21 \mathrm{~min}$ : $80-70 \%$; $21-23 \mathrm{~min}$ : $70 \%$; 23-24 min: 70-95\%; 24-26 min: 95\% (column equilibration). After $15 \mathrm{~min}$, all compounds of interest were eluted; therefore, for the remainder of the analytic run, the eluate was diverged from MS to waste. The ESI source was operated in positive mode and source gas temperature was set to $120^{\circ} \mathrm{C}$, and gas flow to $11 \mathrm{~L} / \mathrm{min}$. The nebulizer pressure was set to $35 \mathrm{psi}$, and the temperature and sheath gas flow was set to $350{ }^{\circ} \mathrm{C}$ and $10 \mathrm{~L} / \mathrm{min}$, respectively. The capillary voltage was set to $4000 \mathrm{~V}$ and the nozzle voltage to $500 \mathrm{~V}$. The dual ion funnel system was set to $210 \mathrm{~V}$ and $160 \mathrm{~V}$, for the high and low-pressure funnel, respectively. Each batch was divided into analytical blocks, consisting of six samples (a blank was injected after the third sample), flanked by a QC low and QC high before and after injection of the samples. Moreover, two blanks were injected in between blocks as well as before and after injection of QCs to avoid carry-over. For semi-quantification of lipids, a dynamic multiple reaction monitoring MS method and a mix of deuterated (deu) PC, LPC and SM internal standards was used; deuPC was used to quantitate PC and PE, whereas deuLPC was used for LPC and LPE, and deuSM was used for SM and Cer. The MS method that was used enables identification at the lipid species level but does not reveal the stereo specific numbering of the fatty acyl residues of the carbon backbone. Thus, the annotation of a glycerophospholipid containing two fatty acyl residues with, e.g., chain lengths of 18 carbons and two double bonds and 18 carbons and one double bond, is written 18:2_18:1, in accordance with the LIPID MAPS consortium [37] nomenclature.

\subsection{LC-MS Data Preprocessing}

LC-MS data processing was performed using the Agilent MassHunter Workstation Software-Data Acquisition for 6400 Series Triple Quadrupole program version B.07.00. A total of 53 phospholipids were annotated. A complete list of the investigated phospholipids is provided in Supplementary Material Table S5. All 1307 plasma samples were analyzed by LC-MS in 41 batches. Each batch included 25-30 real samples, six high concentration quality control mixture samples $(\mathrm{QC})$, and six low concentration $\mathrm{QC}$ samples. Both QC samples, high $Q C$ and low $Q C$, were run in between six real samples within each batch. While within-batch variations of the QC samples were insignificant, larger between-batch variations in the data were observed. Therefore, the LC-MS data were corrected for the between-batch effect using the high QC samples run within batches. The following equation was used for the batch correction:

$$
\mathrm{X}_{\mathrm{COR}(i, j, b)}=\left(\mathrm{X}_{\mathrm{RAW}(i, j, b)} / \operatorname{mean}\left(\mathrm{QC}_{(: ;, j, b)}\right)\right) \times \operatorname{mean}\left(\mathrm{QC}_{(: ;, j, B)}\right)
$$

where, $\mathrm{X}_{\mathrm{COR}}$ is between-batch effect corrected data, $\mathrm{X}_{\mathrm{RAW}}$ is a raw data, $\mathrm{QC}$ is high concentration quality control mixture sample, $i=$ sample, $j=$ variable, $b=$ batch, $B=$ all batches.

According to Equation (1), a peak area of each phospholipid was normalized (divided) by the mean area of the corresponding internal standard analyzed from high QC samples run within the same batch. Then, in order to correct for the magnitude change, normalized peak areas were multiplied by the mean of the corresponding internal standard analyzed from high QC samples run across all batches.

\subsection{Statistical Analysis}

Repeated measurements using a linear mixed model were conducted to investigate meal-time interactions. Overall participant and within-visit participant differences were included as random factors and the analyses were adjusted for BMI, age and visit. Normal probability plots and residuals plots were used to test the assumptions of normal distribution and homogeneity of variance. If the assumptions were not met, data were log-transformed. In order to control for multiple testing, $p$-values were adjusted by the false discovery rate (FDR) using the Benjamini and Hochberg approach [38]. FDR-adjusted 
$p$-values are denominated $q$-values and a $q$-value $<0.05$ was considered significant. When a meal effect was found $(q<0.05)$, differences between the treatment groups were evaluated by Tukey's all-pairwise comparison. One-way ANOVA at time $0 \mathrm{~min}$. revealed no significant differences among treatment groups at the beginning of the postprandial period. All statistical analyses were conducted using the statistical software RStudio (version 1.3.1093).

Supplementary Materials: The following are available online at https: / www.mdpi.com/article / 10.3390/metabo11070454/s1, Table S1: Relative level of ceramide (CER) in plasma samples, Table S2: Relative level of sphingomyelin (SM) in plasma samples, Table S3: Relative level of phosphatidylethanolamine (PE) in plasma samples, Table S4: Relative level of phosphatidylcholine (PC) in plasma samples, Table S5: List of investigated phospholipids, Figure S1: PCA Scores plot, Figure S2: PCA loadings plot, Figure S3: Relative level of phospholipids in plasma samples for (1) LPE 18:2, (2) LPE 18:0, (3) LPE 20:3, (4) LPE 20:2, (5) LPE 20:1, (6) LPC 18:1, (7) LPC 18:0.

Author Contributions: Conceptualization, I.E.I.L., L.K., K.J.J., A.A., M.H., A.R. and H.C.B.; methodology, R.T., I.E.I.L., B.K. and H.C.B.; software, R.T. and B.K.; formal analysis, R.T. and I.E.I.L.; investigation, L.K. and A.R.; resources, M.H. and H.C.B.; data curation, R.T.; writing-original draft preparation, R.T., I.E.I.L. and H.C.B.; writing-review and editing, R.T., H.C.B., I.E.I.L., L.K., K.J.J., A.A., M.H., A.R. and B.K.; project administration, H.C.B., M.H. and A.R.; funding acquisition, M.H. All authors have read and agreed to the published version of the manuscript.

Funding: This research was funded by Arla Food for Health. The intervention products were produced by Arla Innovation Center, Denmark.

Institutional Review Board Statement: The study was conducted according to the guidelines of the Declaration of Helsinki and approved by the regional committee on biomedical research in Denmark (H-18024447). The study was registered at clinicaltrial.gov (NCT03656367).

Informed Consent Statement: Informed consent was obtained from all participants involved in the study. All personal data were handled confidential and stored in accordance with applicable law, General Data Protection Regulation and Danish Data Protection Act. No. 502 of 23 May 2018.

Data Availability Statement: Pseudo-anonymized data can be made available upon request before 2029 via a data sharing contract. From 2029, fully anonymized data can be transferred.

Acknowledgments: The authors thank Kristian Wejse Sanggaard, Arla Food Ingredients, and Emilien Rouy and Mette Bakman, Arla Foods Amba, for their participation in fruitful discussions of the project. We also thank the participants as well as the staff (kitchen staff, laboratory technicians and master thesis students) involved in the trial at the Department of Nutrition, Exercise and Sports, University of Copenhagen.

Conflicts of Interest: R.T., I.E.I.L., B.K., L.K., M.H., A.R. and H.C.B. declare no conflict of interest. K.J.J. is employed by Arla Foods Amba, Denmark. AA is currently employed by the Novo Nordisk Foundation to establish a National Centre for Healthy Weight in Denmark. The funders had no role in the design of the study; in the collection, analyses, or interpretation of data; in the writing of the manuscript, or in the decision to publish the results.

\section{References}

1. Van Staveren, W.A.; de Groot, L.C. Evidence-based dietary guidance and the role of dairy products for appropriate nutrition in the elderly. J. Am. Coll. Nutr. 2011, 30 (Suppl. 1), 429-437. [CrossRef]

2. Lovegrove, J.A. Dietary dilemmas over fats and cardiometabolic risk. Proc. Nutr. Soc. 2020, 79, 11-21. [CrossRef]

3. Astrup, A.; Bertram, H.C.S.; Bonjour, J.-P.; de Groot, L.C.P.; de Oliveira Otto, M.C.; Feeney, E.L.; Garg, M.L.; Givens, I.; Kok, F.J.; Krauss, R.M.; et al. WHO draft guidelines on dietary saturated and trans fatty acids: Time for a new approach? BMJ 2019, 366, 14137. [CrossRef] [PubMed]

4. Feeney, E.L.; Barron, R.; Dible, V.; Hamilton, Z.; Power, Y.; Tanner, L.; Flynn, C.; Bouchier, P.; Beresford, T.; Noronha, N.; et al. Dairy matrix effects: Response to consumption of dairy fat differs when eaten within the cheese matrix-A randomized controlled trial. Am. J. Clin. Nutr. 2018, 108, 667-674. [CrossRef] [PubMed]

5. Soerensen, K.V.; Thorning, T.K.; Astrup, A.; Kristensen, M.; Lorenzen, J.K. Effect of dairy calcium from cheese and milk on fecal fat excretion, blood lipids, and appetite in young men. Am. J. Clin. Nutr. 2014, 99, 984-991. [CrossRef]

6. Hjerpsted, J.; Leedo, E.; Tholstrup, T. Cheese intake in large amounts lowers LDL-cholesterol concentrations compared with butter intake of equal fat content. Am. J. Clin. Nutr. 2011, 94, 1479-1484. [CrossRef] [PubMed] 
7. Thorning, T.K.; Bertram, H.C.; Bonjour, J.-P.; De Groot, L.; Dupont, D.; Feeney, E.; Ipsen, R.; Lecerf, J.M.; Mackie, A.; McKinley, M.C.; et al. Whole dairy matrix or single nutrients in assessment of health effects: Current evidence and knowledge gaps. Am. J. Clin. Nutr. 2017, 105, 1033-1045. [CrossRef]

8. Fardet, A.; Dupont, D.; Rioux, L.-E.; Turgeon, S.L. Influence of food structure on dairy protein, lipid and calcium bioavailability: A narrative review of evidence. Crit. Rev. Food Sci. Nutr. 2019, 59, 1987-2010. [CrossRef]

9. Lorenzen, J.K.; Astrup, A. Dairy calcium intake modifies responsiveness of fat metabolism and blood lipids to a high-fat diet. Br. J. Nutr. 2011, 105, 1823-1831. [CrossRef] [PubMed]

10. Lorenzen, J.K.; Jensen, S.K.; Astrup, A. Milk minerals modify the effect of fat intake on serum lipid profile: Results from an animal and a human short-term study. Br. J. Nutr. 2014, 111, 1412-1420. [CrossRef]

11. Ayala-Bribiesca, E.; Turgeon, S.L.; Britten, M. Effect of calcium on fatty acid bioaccessibility during in vitro digestion of Cheddartype cheeses prepared with different milk fat fractions. J. Dairy Sci. 2017, 100, 2454-2470. [CrossRef]

12. Ayala-Bribiesca, E.; Turgeon, S.L.; Pilon, G.; Marette, A.; Britten, M. Postprandial lipemia and fecal fat excretion in rats is affected by the calcium content and type of milk fat present in Cheddar-type cheeses. Food Res. Int. 2018, 107, 589-595. [CrossRef]

13. Torcello-Gómez, A.; Boudard, C.; Mackie, A.R. Calcium Alters the Interfacial Organization of Hydrolyzed Lipids during Intestinal Digestion. Langmuir 2018, 34, 7536-7544. [CrossRef] [PubMed]

14. Vors, C.L.; Joumard-Cubizolles, M.; Lecomte, E.; Combe, L.; Ouchchane, J.; Drai, K.; Raynal, F.; Joffre, L.; Meiller, M.; Le Barz, P.; et al. Milk polar lipids reduce lipid cardiovascular risk factors in overweight postmenopausal women: Towards a gut sphingomyelin-cholesterol interplay. Gut 2020, 69, 487-501. [CrossRef] [PubMed]

15. Noh, S.K.; Koo, S.I. Milk Sphingomyelin Is More Effective than Egg Sphingomyelin in Inhibiting Intestinal Absorption of Cholesterol and Fat in Rats. J. Nutr. 2004, 134, 2611-2616. [CrossRef] [PubMed]

16. Wat, E.; Tandy, S.; Kapera, E.; Kamili, A.; Chung, R.W.; Brown, A.; Rowney, M.; Cohn, J.S. Dietary phospholipid-rich dairy milk extract reduces hepatomegaly, hepatic steatosis and hyperlipidemia in mice fed a high-fat diet. Atherosclerosis 2009, 205, 144-150. [CrossRef]

17. Kamili, A.; Wat, E.; Chung, R.W.; Tandy, S.; Weir, J.M.; Meikle, P.J.; Cohn, J.S. Hepatic accumulation of intestinal cholesterol is decreased and fecal cholesterol excretion is increased in mice fed a high-fat diet supplemented with milk phospholipids. Nutr. Metab. 2010, 7, 90. [CrossRef]

18. LeComte, M.; Bourlieu, C.; Fouilloux-Meugnier, E.; Penhoat, A.; Cheillan, D.; Pineau, G.; Loizon, E.; Trauchessec, M.; Claude, M.; Ménard, O.; et al. Milk Polar Lipids Affect In Vitro Digestive Lipolysis and Postprandial Lipid Metabolism in Mice. J. Nutr. 2015, 145, 1770-1777. [CrossRef]

19. Lindahl, I.E.I.; Artegoitia, V.M.; Downey, E.; O'Mahony, J.A.; O'Shea, C.-A.; Ryan, C.A.; Kelly, A.L.; Bertram, H.C.; Sundekilde, U.K. Quantification of Human Milk Phospholipids: The Effect of Gestational and Lactational Age on Phospholipid Composition. Nutrients 2019, 11, 222. [CrossRef]

20. Kjølbæk, L.; Schmidt, J.M.; Rouy, E.; Jensen, K.J.; Astrup, A.; Bertram, H.C.; Hammershøj, M.; Raben, A. Matrix structure of cheese products results in different postprandial lipid responses: A randomized, crossover trial. Am. J. Clin. Nutr. 2021. accepted for publication. [CrossRef]

21. Schmidt, J.M.; Kjølbæk, L.; Jensen, K.J.; Rouy, E.; Bertram, H.C.; Larsen, T.; Raben, A.; Astrup, A.; Hammershøj, M. Influence of type of dairy matrix micro- and macrostructure on in vitro lipid digestion. Food Funct. 2020, 11, 4960-4972. [CrossRef]

22. Drouin-Chartier, J.-P.; Tremblay, A.J.; Maltais-Giguère, J.; Charest, A.; Guinot, L.; Rioux, L.-E.; Labrie, S.; Britten, M.; Lamarche, B.; Turgeon, S.L.; et al. Differential impact of the cheese matrix on the postprandial lipid response: A randomized, crossover, controlled trial. Am. J. Clin. Nutr. 2017, 106, 1358-1365. [CrossRef]

23. Anto, L.; Warykas, S.W.; Torres-Gonzalez, M.; Blesso, C.N. Milk Polar Lipids: Underappreciated Lipids with Emerging Health Benefits. Nutrients 2020, 12, 1001. [CrossRef] [PubMed]

24. Contarini, G.; Povolo, M. Phospholipids in Milk Fat: Composition, Biological and Technological Significance, and Analytical Strategies. Int. J. Mol. Sci. 2013, 14, 2808-2831. [CrossRef] [PubMed]

25. Mazzei, J.C.; Zhou, H.; Brayfield, B.P.; Hontecillas, R.; Bassaganya-Riera, J.; Schmelz, E.M. Suppression of intestinal inflammation and inflammation-driven colon cancer in mice by dietary sphingomyelin: Importance of peroxisome proliferator-activated receptor $\gamma$ expression. J. Nutr. Biochem. 2011, 22, 1160-1171. [CrossRef]

26. Norris, G.H.; Jiang, C.; Ryan, J.; Porter, C.M.; Blesso, C.N. Milk sphingomyelin improves lipid metabolism and alters gut microbiota in high fat diet-fed mice. J. Nutr. Biochem. 2016, 30, 93-101. [CrossRef]

27. Averill, M.; Rubinow, K.B.; Cain, K.; Wimberger, J.; Babenko, I.; Becker, J.O.; Foster-Schubert, K.E.; Cummings, D.E.; Hoofnagle, A.N.; Vaisar, T. Postprandial remodeling of high-density lipoprotein following high saturated fat and high carbohydrate meals. $J$. Clin. Lipidol. 2020, 14, 66-76.e11. [CrossRef] [PubMed]

28. Meikle, P.J.; Barlow, C.; Mellett, N.A.; Mundra, P.A.; Bonham, M.; Larsen, E.A.; Cameron-Smith, D.; Sinclair, A.J.; Nestel, P.J.; Wong, G. Postprandial Plasma Phospholipids in Men Are Influenced by the Source of Dietary Fat. J. Nutr. 2015, 145, 2012-2018. [CrossRef]

29. Chatelaine, H.; Dey, P.; Mo, X.; Mah, E.; Bruno, R.S.; Kopec, R.E. Vitamin A and D Absorption in Adults with Metabolic Syndrome versus Healthy Controls: A Pilot Study Utilizing Targeted and Untargeted LC-MS Lipidomics. Mol. Nutr. Food Res. 2021, 65, e2000413. [CrossRef] [PubMed] 
30. Weiland, A.; Bub, A.; Barth, S.W.; Schrezenmeir, J.; Pfeuffer, M. Effects of dietary milk- and soya-phospholipids on lipidparameters and other risk indicators for cardiovascular diseases in overweight or obese men-Two double-blind, randomised, controlled, clinical trials. J. Nutr. Sci. 2016, 5, e21. [CrossRef]

31. Hussain, M.M. Intestinal lipid absorption and lipoprotein formation. Curr. Opin. Lipidol. 2014, 25, 200-206. [CrossRef]

32. Sanggaard, K.M.; Holst, J.J.; Rehfeld, J.F.; Sandström, B.; Raben, A.; Tholstrup, T. Different effects of whole milk and a fermented milk with the same fat and lactose content on gastric emptying and postprandial lipaemia, but not on glycaemic response and appetite. Br. J. Nutr. 2004, 92, 447-459. [CrossRef]

33. Ehrlein, H.-J.; Pröve, J. Effect of viscosity of test meals on gastric emptying in dogs. Q. J. Exp. Physiol. 1982, 67, 419-425. [CrossRef] [PubMed]

34. Armand, M.; Pasquier, B.; André, M.; Borel, P.; Senft, M.; Peyrot, J.; Salducci, J.; Portugal, H.; Jaussan, V.; Lairon, D. Digestion and absorption of 2 fat emulsions with different droplet sizes in the human digestive tract. Am. J. Clin. Nutr. 1999, 70, 1096-1106. [CrossRef] [PubMed]

35. Jukkola, A.; Rojas, O.J. Milk fat globules and associated membranes: Colloidal properties and processing effects. Adv. Colloid Interface Sci. 2017, 245, 92-101. [CrossRef] [PubMed]

36. Bligh, E.G.; Dyer, W.J. A rapid method of total lipid extraction and purification. Can. J. Biochem. Physiol. 1959, 37, 911-917. [CrossRef]

37. Liebisch, G.; Vizcaino, J.A.; Köfeler, H.; Trötzmüller, M.; Griffiths, W.; Schmitz, G.; Spener, F.; Wakelam, M. Shorthand notation for lipid structures derived from mass spectrometry. J. Lipid Res. 2013, 54, 1523-1530. [CrossRef]

38. Benjamini, Y.; Hochberg, Y. Controlling the False Discovery Rate-A Practical and Powerful Approach to Multiple Testing. J. R. Stat. Soc. Ser. B 1995, 57, 289-300. [CrossRef] 